\title{
Toeplitz-type operators in weighted Morrey spaces
}

\author{
Peizhu Xie ${ }^{1,2^{*}}$ and Guangfu Cao ${ }^{1,2}$
}

${ }^{\text {*Correspondence: }}$

xiepeizhu82@163.com

'school of Mathematics and Information Science, Guangzhou

University, Guangzhou, 510006,

China

${ }^{2}$ Key Laboratory of Mathematics and Interdisciplinary Sciences of Guangdong Higher Education Institutes, Guangzhou University, Guangzhou, 510006, China

\begin{abstract}
Let $T_{j, 1}$ and $T_{j, 2}$ be singular integrals with non-smooth kernels, which are associated with an approximation of identity or \pm / ( / is the identity operator). Denote the Toeplitz-type operator by $T_{b}=\sum_{j=1}^{N} T_{j, 1} M_{b} T_{j, 2}$, where $M_{b} f(x)=b(x) f(x)$. In this paper, the estimates of the Toeplitz operator $T_{b}(f)$ related to singular integral operators with non-smooth kernels and $b \in B M O\left(\mathbb{R}^{n}\right)$ in weighted Morrey spaces is established.
\end{abstract}

MSC: $47 \mathrm{~B} 35$

Keywords: Toeplitz operator; Morrey space; $A_{p}$ weights

\section{Introduction}

The classical Morrey spaces were introduced by Morrey in [1] to investigate the local behavior of solutions to second-order elliptic partial differential equations. The boundedness of the Hardy-Littlewood maximal operator, the singular integral operator, the fractional integral operator and the commutator of these operators in Morrey spaces have been studied by many authors; see [2-5] and the references therein. In [6], Komori and Shirai studied the boundedness of these operators in weighted spaces.

It is well known that the commutator $[b, T]$ is defined by $[b, T](f)=T(b f)-b T(f)$, where $T$ is a Calderón-Zygmund operator and $b \in B M O$. The commutator generated by the Calderón-Zygmund operators and a locally integrable function $b$ can be regarded as a special case of the Toeplitz operator $T_{b}=\sum_{j=1}^{N} T_{j, 1} M_{b} T_{j, 2}$, where $T_{j, 1}$ and $T_{j, 2}$ are the CalderónZygmund operators or $\pm I$ ( $I$ is the identity operator), $M_{b} f(x)=b(x) f(x)$. When $b \in B M O$, Krantz and Li discussed the $L^{p}$ boundedness of $T_{b}$ on the homogeneous space, see [7, 8]. In [9], the authors studied the boundedness of $T_{b}$ in Morrey spaces. In this paper, we study the boundedness of Toeplitz-type operators related to singular integral operators with non-smooth kernels in weighted Morrey spaces.

The singular integral operators with non-smooth kernels previously appeared in [10]. We say that $T$ is a singular integral operator with non-smooth kernel if it satisfies the following conditions.

(i) There exists a class of operators $A_{t}$ with kernels $a_{t}(x, y)$, which satisfy the condition (2.3) in Section 2, so that the kernels $k_{t}(x, y)$ of the operators $\left(T-A_{t} T\right)$ satisfy the condition

$$
\left|k_{t}(x, y)\right| \leq c \frac{t^{\gamma / m}}{|x-y|^{n+\gamma}}
$$

when $|x-y| \geq c_{1} t^{1 / m}$ for some $\gamma, m>0$. 
(ii) There exists a class of operators $B_{t}$ with kernels $b_{t}(x, y)$, which satisfy the condition (2.3), such that $\left(T-T B_{t}\right)$ have associated kernels $K_{t}(x, y)$ and there exist positive constants $c_{2}, c_{3}$ such that

$$
\int_{|x-y| \geq c_{2} t^{1 / m}}\left|K_{t}(x, y)\right| d x \leq c_{3} \quad \text { for all } y \in \mathbb{R}^{n}
$$

Note that the classes of operators $A_{t}$ and $B_{t}$ play the role of a generalized approximation to the identity. It is not difficult to check that conditions (1.1) and (1.2) are consequences of the standard Calderón-Zygmund operator. See Proposition 2 in [10].

The paper is organized as follows. In Section 2, we recall some important estimates on BMO functions, maximal functions and sharp maximal functions. In Section 3, we prove the main result.

\section{Definitions and preliminary results}

Let $1 \leq p<\infty, 0<\kappa<1$ and $w$ be a weight. The weighted Morrey space is defined by

$$
L^{p, \kappa}(w):=\left\{f \in L_{\mathrm{loc}}^{p}(w):\|f\|_{L^{p, \kappa}(w)}<\infty\right\}
$$

where

$$
\|f\|_{L^{p, \kappa}(w)}=\sup _{B}\left(\frac{1}{w(B)^{\kappa}} \int_{B}|f|^{p} w d x\right)^{1 / p},
$$

and the supremum is taken over all balls $B$ in $\mathbb{R}^{n}$. If $w=1$ and $\kappa=\lambda / n$ with $0<\lambda<n$, then $L^{p, \kappa}(w)=L^{p, \lambda}\left(\mathbb{R}^{n}\right)$, the classical Morrey spaces.

The standard Hardy-Littlewood maximal function $M_{r} f, 1 \leq r<\infty$, is defined by

$$
M_{r} f(x)=\sup _{B: x \in B}\left(\frac{1}{|B|} \int_{B}|f(y)|^{r} d y\right)^{1 / r},
$$

where the sup is taken over all balls containing $x$. If $r=1, M_{1} f$ will be denoted by $M f$. The Fefferman-Stein sharp maximal function of $f, f^{\sharp}(x)$, is defined by

$$
f^{\sharp}(x)=\sup _{B: x \in B} \frac{1}{|B|} \int_{B}\left|f(y)-f_{B}\right| d y,
$$

where $f_{B}=\frac{1}{|B|} \int_{B} f d x$. We will say $f \in B M O\left(\mathbb{R}^{n}\right)$ if $f \in L_{\text {loc }}^{1}\left(\mathbb{R}^{n}\right)$ and $f^{\sharp}(x) \in L^{\infty}$. If $f \in B M O$, the $B M O$ semi-norm of $f$ is given by

$$
\|f\|_{*}=\sup _{x} f^{\sharp}(x)=\sup _{x} \sup _{x \in B} \frac{1}{|B|} \int_{B}\left|f(y)-f_{B}\right| d y .
$$

A weight $w$ is a non-negative locally integrable function. We say that $w \in A_{p}\left(\mathbb{R}^{n}\right), 1<p<$ $\infty$, if there exists a constant $C$ such that for every ball $B \subset \mathbb{R}^{n}$,

$$
\left(\frac{1}{|B|} \int_{B} w d x\right)\left(\frac{1}{|B|} \int_{B} w^{1-p^{\prime}} d x\right)^{p-1} \leq C,
$$


where $\frac{1}{p}+\frac{1}{p^{\prime}}=1$. For $p=1$, we say that $w \in A_{1}\left(\mathbb{R}^{n}\right)$ if there is a constant $C$ such that for every ball $B \subset \mathbb{R}^{n}$,

$$
\frac{1}{|B|} \int_{B} w d y \leq C w(x) \text { for a.e. } x \in B
$$

or, equivalently, $M(w) \leq C w$ a.e. We denote $A_{\infty}\left(\mathbb{R}^{n}\right)=\bigcup_{1 \leq p<\infty} A_{p}\left(\mathbb{R}^{n}\right)$. For the above definition, see [11].

A family of operators $A_{t}, t>0$, is said to be a 'generalized approximation to the identity' if, for every $t>0, A_{t}$ can be represented by kernels $a_{t}(x, y)$ in the following sense: For every function $f \in L^{p}\left(\mathbb{R}^{n}\right), p \geq 1, A_{t} f(x)=\int_{\mathbb{R}^{n}} a_{t}(x, y) f(y) d y$, and the following condition holds:

$$
\left|a_{t}(x, y)\right| \leq h_{t}(x, y)=t^{-n / m} s\left(|x-y|^{m} t^{-1}\right)
$$

in which $m$ is a positive constant and $s$ is a positive, bounded, decreasing function satisfying

$$
\lim _{r \rightarrow \infty} r^{n+N+\epsilon} s\left(r^{m}\right)=0
$$

for some $\epsilon>0$.

Note that (2.2) implies that

$$
\left|a_{t}(x, y)\right| \leq t^{-n / m} \times\left(1+\frac{|x-y|}{t^{1 / m}}\right)^{-(n+\epsilon)} .
$$

In [12], the sharp maximal function $M_{A}^{\sharp} f$ associated with a 'generalized approximation to the identity' $\left\{A_{t}, t>0\right\}$ is defined by

$$
M_{A}^{\sharp} f(x)=\sup _{x \in B} \frac{1}{|B|} \int_{B}\left|f(y)-A_{t_{B}} f(y)\right| d y,
$$

where $t_{B}=r_{B}^{m}$, and $f \in L^{p}\left(\mathbb{R}^{n}\right)$ for some $p \geq 1$.

The following results are proved in the context of spaces of homogeneous type in [13, 14] and [10].

\section{Lemma 2.1}

(i) For every $p \in[1, \infty)$, there exists a constant $C$ such that for every $f \in L^{p}\left(\mathbb{R}^{n}\right)$,

$$
A_{t} f(x) \leq C M f(x)
$$

(ii) Assume that $b \in B M O$ and $M>1$. Then, for every ball $B(x ; r)$, we have

$$
\left|b_{B}-b_{M B}\right| \leq C\|b\|_{*} \log M
$$

(iii) (John-Nirenberg lemma) Let $1 \leq p<\infty$ and $B \subset \mathbb{R}^{n}$, then $b \in B M O$ if and only if

$$
\frac{1}{|B|} \int_{B}\left|b-b_{B}\right|^{p} d x \leq\|b\|_{*}^{p} .
$$


Lemma 2.2 For $1<p<\infty, 0<\kappa<1$ and $w \in A_{p}$, we have $\|M f\|_{L^{p, \kappa}(w)} \leq C\|f\|_{L^{p, \kappa}(w)}$.

For the proof of this lemma, see [2, Theorem 3.2].

Lemma 2.3 Let $\left\{A_{t}, t>0\right\}$ be a 'generalized approximation to the identity' and let $b \in$ BMO. Then, for every function $f \in L^{p}\left(\mathbb{R}^{n}\right), p>1, x \in \mathbb{R}^{n}$ and $1<r<\infty$, we have

$$
\sup _{x \in B} \frac{1}{|B|} \int_{B}\left|A_{t_{B}}\left(b-b_{B}\right) f(y)\right| d y \leq C\|b\|_{*} M_{r} f(x)
$$

where $t_{B}=r_{B}^{m}$.

For the proof of this lemma, see Lemma 2.3 in [15].

Now, we have the following analogy of the classical Fefferman-Stein inequality [11, Chapter IV] for the sharp maximal function $M_{A}^{\sharp} f$. For the proof, see Proposition 4.1 in [12].

Lemma 2.4 Take $\lambda>0, w \in A_{\infty}\left(\mathbb{R}^{n}\right), f \in L_{\text {loc }}^{1}$ and a ball $B_{0}$ such that there exists $x_{0} \in B_{0}$ with $M f\left(x_{0}\right)<\lambda$. Then, for every $0<\eta<1$, there exist $r, \gamma>0$ (independent of $\lambda, B_{0}, f, x_{0}$ ) and $C_{w}$ which only depend on $w$ such that

$$
w\left\{x \in B_{0}: M f(x)>D \lambda, M_{A}^{\sharp} f(x) \leq \gamma \lambda\right\} \leq C_{w} \eta^{r} w\left(B_{0}\right),
$$

where $D>1$ is a fixed constant which depends only on the 'generalized approximation to the identity' $\left\{A_{t}, t>0\right\}$.

\section{The main results}

In this section, we consider the Toeplitz operator related to a singular integral with nonsmooth kernel $T_{b}=\sum_{j=1}^{M} T_{j, 1} M_{b} T_{j, 2}$, where $T_{j, 1}$ and $T_{j, 2}$ are singular integrals with nonsmooth kernels, which are associated with an approximation of identity or $\pm I$. For $i=$ $1, \ldots, M, j=1,2$, we assume that if $T_{i, j} \neq \pm I$, then:

(a) $T_{i, j}$ are bounded operators on $L^{2}\left(\mathbb{R}^{n}\right)$.

(b) There exist 'generalized approximations of the identity' $\left\{B_{t}^{i j}, t>0\right\}$ such that $\left(T_{i, j}-T_{i, j} B_{t}^{i j}\right)$ have associated kernels $K_{t}^{i j}(x, y)$ and there exist positive constants $C_{1}$, $C_{2}$ such that

$$
\int_{|x-y|>C_{1} t^{1 / m}}\left|K_{t}^{i j}(x, y)\right| d x \leq C_{2} \quad \text { for all } y \in \mathbb{R}^{n}
$$

(c) There exists a 'generalized approximation to the identity' $\left\{A_{t}, t>0\right\}$ such that the kernels $k_{t}^{i j}(x, y)$ of the operators $\left(T_{i, j}-A_{t} T_{i, j}\right)$ satisfy

$$
\left|k_{t}^{i j}\right| \leq C_{4} \frac{t^{\alpha / m}}{|x-y|^{n+\alpha}} \frac{t^{\alpha / m}}{d(x, y)^{\alpha}},
$$

when $|x-y| \geq C_{3} t^{1 / m}$ for some $C_{3}, C_{4}, \alpha>0$. 
It is proved in [10] that if $T$ is an operator satisfying (a) and (b) above, then $T$ is of weak $(1,1)$ and of strong type $(p, p)$ for $1<p \leq 2$. In addition, if (c) is also satisfied, the operator $T$ is bounded on $L^{p}\left(\mathbb{R}^{n}\right)$ for all $1<p<\infty$. Moreover, if $w \in A_{p}$, then $T$ is bounded on $L^{p}(w)$ (see [12]).

In order to study the boundedness of $T_{b}$ in weighted Morrey spaces, we need the following result.

Lemma 3.1 Let $w \in A_{\infty}, 0<\kappa<1$ and $1<p<\infty$. Then, for everyf $\in L_{\text {loc }}^{1}$ with $M f \in L^{p, \kappa}(w)$, there exists a constant $C_{w}$, which only depends on $w$, such that

$$
\|M f\|_{L^{p, \kappa}(w)} \leq C_{w}\left\|M_{A}^{\sharp} f\right\|_{L^{p, \kappa}(w)} .
$$

Proof Let $B$ be a ball in $\mathbb{R}^{n}$. Set $E_{\lambda}=\{x \in B: M f(x)>\lambda\}$. Then from the Whitney decomposition theorem, we know that there exist mutually disjoint cubes $Q_{k}$ such that $E_{\lambda}=\bigcup_{k} Q_{k}$ and $10 Q_{k} \cap B \backslash E_{\lambda} \neq \varnothing$. Denote $B_{k}$ to be the ball with the same center as $Q_{k}$ and $r_{B_{k}}=\frac{1}{2}$ diameter $Q_{k}$. Let $\widetilde{B}_{k}=10 B_{k}$. Then there exists an $x_{k} \in \widetilde{B}_{k} \cap B \backslash E_{\lambda}$, that is, $M f\left(x_{k}\right) \leq \lambda$. Let us use Lemma 2.4. There are $C_{w} ; r>0$ and $D>1$ such that, if $0<\eta<1$ (to be chosen later), we can find $\gamma>0$ in such a way that

$$
w\left\{x \in \widetilde{B}_{k}: M f(x)>D \lambda, M_{A}^{\sharp} f(x) \leq \gamma \lambda\right\} \leq C_{w} \eta^{r} w\left(\widetilde{B}_{k}\right) .
$$

Set $U_{\lambda}=\left\{x \in B: M f(x)>D \lambda, M_{A}^{\sharp} f(x) \leq \gamma \lambda\right\}$ and so $U_{\lambda} \subset E_{\lambda}=\bigcup_{k} Q_{k} \subset \bigcup_{k} \widetilde{B}_{k}$ since $D>1$. Then

$$
\begin{aligned}
w\left(U_{\lambda}\right) & \leq \sum_{k} w\left\{x \in \widetilde{B}_{k}: M f(x)>D \lambda, M_{A}^{\sharp} f(x) \leq \gamma \lambda\right\} \\
& \leq C \eta^{r} \sum_{k} w\left(\widetilde{B}_{k}\right) \\
& \leq C \eta^{r} \sum_{k} w\left(Q_{k}\right)=C \eta^{r} w\left(E_{\lambda}\right) \\
& =C \eta^{r} w\{x \in B: M f(x)>\lambda\},
\end{aligned}
$$

where we used the fact that $A_{\infty}$ weights are doubling measures and $C$ is a constant that only depends on the weight. One can prove that

$$
\begin{aligned}
\int_{B}|M f|^{p} w d x & =D^{p} \int_{0}^{\infty} p \lambda^{p-1} w\{x \in B: M f(x)>D \lambda\} d \lambda \\
& \leq D^{p} \int_{0}^{\infty} p \lambda^{p-1}\left(w\left(U_{\lambda}\right)+w\left\{x \in B: M_{A}^{\sharp} f(x)>\gamma \lambda\right\}\right) d \lambda \\
& \leq C D^{p} \eta^{r} \int_{B}|M f|^{p} w d x+\frac{D^{p}}{\gamma^{p}} \int_{B}\left|M_{A}^{\sharp} f\right|^{p} w d x .
\end{aligned}
$$

Let us choose $\eta$ such that $C D^{p} \eta^{r}=1 / 2$. The former inequality turns out to be

$$
\int_{B}|M f|^{p} w d x \leq 2 \frac{D^{p}}{\gamma^{p}} \int_{B}\left|M_{A}^{\sharp} f\right|^{p} w d x .
$$


This implies that

$$
\|M f\|_{L^{p, \kappa}(w)} \leq C\left\|M_{A}^{\sharp} f\right\|_{L^{p, \kappa}(w)} .
$$

The proof of this lemma is completed.

The aim of this section is to prove the following theorem.

Theorem 3.2 Let $T_{i, j}$ be operators satisfying the above conditions (a), (b) and (c) or $\pm I$. Let $1<p<\infty, 0<\kappa<1$ and $w \in A_{p}$. Suppose that $T_{1}(f)=0$ when $f \in L^{p, \kappa}(w)$. If $b \in B M O\left(\mathbb{R}^{n}\right)$, then there exists a constant $C$ such that

$$
\left\|T_{b}(f)\right\|_{L^{p, \kappa}(w)} \leq C\left(\sum_{j=1}^{M}\left\|T_{j, 1}\right\|_{L^{2}\left(\mathbb{R}^{n}\right)}\right)\left(\sum_{j=1}^{M}\left\|T_{j, 2}\right\|_{L^{2}\left(\mathbb{R}^{n}\right)}\right)\|b\|_{*}\|f\|_{L^{p, \kappa}(w)}
$$

for all $f \in L^{p, \kappa}(w)$.

Proof Without loss of generality, we may assume that

$$
\left\|T_{j, i}\right\|_{L^{2}\left(\mathbb{R}^{n}\right)} \leq 1 \quad \text { for all } 1 \leq j \leq M, i=1,2
$$

For $w \in A_{p}$, it is well known that there exists $t>1$ such that $w \in A_{p / t}$. Then we can choose two real numbers $r$ and $s$ larger than 1 such that $1<r s<p<\infty$ and $w \in A_{p /(r s)}$. We will prove that there exists a constant $C$ such that

$$
M_{A}^{\sharp}\left(T_{b} f\right)(x) \leq \sum_{j=1}^{M} C\|b\|_{*} M_{r s}\left(T_{j, 2} f\right)(x)
$$

for all $x \in \mathbb{R}^{n}$.

We now prove (3.4). For an arbitrary fixed $x \in \mathbb{R}^{n}$, choose a ball $B\left(x_{0} ; r\right)=\left\{y \in \mathbb{R}^{n}: \mid x_{0}-\right.$ $y \mid<r\}$ which contains $x$. We have that $T_{1}(f)=0$, and so $T_{b_{B}}(f)=b_{B} T_{1}(f)=0$. Thus

$$
T_{b}(f)=T_{\left(b-b_{B}\right) \chi_{2 B}}(f)+T_{\left(b-b_{B}\right) \chi_{(2 B)}}(f)
$$

and

$$
A_{t_{B}}\left(T_{b} f\right)=A_{t_{B}}\left(T_{\left(b-b_{B}\right) \chi_{2 B}} f\right)+A_{t_{B}}\left(T_{\left(b-b_{B}\right) \chi_{(2 B)} c} f\right),
$$

where $t_{B}=r_{B}^{m}$. Then

$$
\begin{aligned}
\frac{1}{|B|} \int_{B}\left|T_{b}(f)(y)-A_{t_{B}}\left(T_{b} f\right)(y)\right| d y \\
\leq \frac{1}{|B|} \int_{B}\left|T_{\left(b-b_{B}\right) \chi_{2 B}}(f)(y)\right| d y+\frac{1}{|B|} \int_{B}\left|A_{t_{B}}\left(T_{\left(b-b_{B}\right) \chi_{2 B}} f\right)(y)\right| d y \\
\quad+\frac{1}{|B|} \int_{B}\left|T_{\left(b-b_{B}\right) \chi_{(2 B)}}(f)(y)-A_{t_{B}}\left(T_{\left(b-b_{B}\right) \chi_{(2 B)}} f\right)(y)\right| d y \\
=: I+I I+I I I .
\end{aligned}
$$


Let $r^{\prime}$ be the dual of $r$ such that $1 / r+1 / r^{\prime}=1$. By Lemma 2.1 and the boundedness of $T_{j, 1}$, we have

$$
\begin{aligned}
I & \leq\left(\frac{1}{|B|} \int_{\mathbb{R}^{n}}\left|T_{\left(b-b_{B}\right) \chi_{2 B}}(f)(y)\right|^{s} d y\right)^{1 / s} \\
& \leq \sum_{j=1}^{M} C\left(\frac{1}{|B|} \int_{2 B}\left|\left(b(y)-b_{B}\right) T_{j, 2}(f)(y)\right|^{s} d y\right)^{1 / s} \\
& \leq \sum_{j=1}^{M} C\left(\frac{1}{|B|} \int_{2 B}\left|b(y)-b_{B}\right|^{s r^{\prime}} d y\right)^{1 /\left(s r^{\prime}\right)}\left(\frac{1}{|B|} \int_{2 B}\left|T_{j, 2}(f)(y)\right|^{s r} d y\right)^{1 /(s r)} \\
& \leq \sum_{j=1}^{M} C\|b\|_{*} M_{r s}\left(T_{j, 2} f\right)(x) .
\end{aligned}
$$

Similarly, by Lemma 2.1 and the boundedness of $T_{j, 1}$, we obtain

$$
\begin{aligned}
I I & \leq \frac{1}{|B|} \int_{B}\left|M\left(T_{\left(b-b_{B}\right) \chi_{2 B}} f\right)(y)\right| d y \leq\left(\frac{1}{|B|} \int_{\mathbb{R}^{n}}\left|M\left(T_{\left(b-b_{B}\right) \chi_{2 B}} f\right)(y)\right|^{s} d y\right)^{1 / s} \\
& \leq\left(\frac{1}{|B|} \int_{\mathbb{R}^{n}}\left|T_{\left(b-b_{B}\right) \chi_{2 B}}(f)(y)\right|^{s} d y\right)^{1 / s} \\
& \leq \sum_{j=1}^{M} C\|b\|_{*} M_{r s}\left(T_{j, 2} f\right)(x) .
\end{aligned}
$$

We now consider the term III. There are two cases:

(1) Suppose that $T_{j, 1} \neq \pm I(j=1, \ldots, M)$, then using the assumption (c), we have

$$
\begin{aligned}
I I I \leq & \sum_{j=1}^{M} \frac{1}{|B|} \int_{B} \int_{(2 B)^{c}}\left|k_{t_{B}}^{j, 1}(y, z)\right|\left|\left(b(z)-b_{B}\right) T_{j, 2}(f)(z)\right| d z d y \\
\leq & C \sum_{j=1}^{M} \sum_{k=1}^{\infty} \int_{2^{k} r_{B} \leq\left|x_{0}-z\right|<2^{k+1} r_{B}} \frac{r_{B}^{\alpha}}{\left|x_{0}-z\right|^{n+\alpha}}\left|\left(b(z)-b_{B}\right) T_{j, 2}(f)(z)\right| d z \\
\leq & C \sum_{j=1}^{M} \sum_{k=1}^{\infty} 2^{-k \alpha} \frac{1}{\left|B\left(x_{0} ; 2^{k} r_{B}\right)\right|} \int_{\left|x_{0}-z\right|<2^{k+1} r_{B}}\left|\left(b(z)-b_{B}\right) T_{j, 2}(f)(z)\right| d z \\
\leq & C \sum_{j=1}^{M} \sum_{k=1}^{\infty} 2^{-k \alpha} \frac{1}{\left|B\left(x_{0} ; 2^{k} r_{B}\right)\right|} \int_{\left|x_{0}-z\right|<2^{k+1} r_{B}}\left|b(z)-b_{2^{k+1} B}\right|\left|T_{j, 2}(f)(z)\right| d z \\
& +C \sum_{j=1}^{M} \sum_{k=1}^{\infty} 2^{-k \alpha}\left|b_{2^{k+1} B}-b_{B}\right| \frac{1}{\left|B\left(x_{0} ; 2^{k} r_{B}\right)\right|} \int_{\left|x_{0}-z\right|<2^{k+1} r_{B}}\left|T_{j, 2}(f)(z)\right| d z \\
\leq & C\|b\|_{*} \sum_{j=1}^{M} \sum_{k=1}^{\infty} 2^{-k \alpha} M_{r s}\left(T_{j, 2} f\right)(x)+C\|b\|_{*} \sum_{j=1}^{M} \sum_{k=1}^{\infty} 2^{-k \alpha}(k+1) M\left(T_{j, 2} f\right)(x) \\
\leq & C\|b\|_{*} \sum_{j=1}^{M} M_{r s}\left(T_{j, 2} f\right)(x) .
\end{aligned}
$$


(2) Suppose that there are $i$ identity or $-I$ operators in $\left\{T_{j, 1}\right\}$. Without loss of generality, we assume that $T_{1,1}, \ldots, T_{i, 1}$ are identity operators, then

$$
\begin{aligned}
I I I \leq & \sum_{j=1}^{i} \frac{1}{|B|} \int_{B}\left|\left(b(z)-b_{B}\right) \chi_{(2 B)^{c}} T_{j, 2}(f)(z)\right| d z \\
& +\sum_{j=1}^{i} \frac{1}{|B|} \int_{B}\left|A_{t_{B}}\left(\left(b-b_{B}\right) \chi_{(2 B)^{c}} T_{j, 2}(f)\right)(z)\right| d z \\
& +\sum_{j=i+1}^{M} \frac{1}{|B|} \int_{B}\left|T_{j, 1} M_{\left(b-b_{B}\right) \chi_{(2 B)^{c}}} T_{j, 2}(f)(z)-A_{t_{B}}\left(T_{j, 1} M_{\left(b-b_{B}\right) \chi_{(2 B)^{c}}} T_{j, 2}(f)\right)(z)\right| d z \\
= & I I I_{1}+I I I_{2}+I I I_{3} .
\end{aligned}
$$

It is obvious that $I I I_{1}=0$.

By (2.4) and Lemma 2.1, we obtain

$$
\begin{aligned}
I I I_{2} & \leq \sum_{j=1}^{i} \frac{1}{|B|} \int_{B} \int_{(2 B)^{c}}\left|h_{t_{B}}(z, y)\left(b(y)-b_{B}\right) T_{j, 2}(f)(y)\right| d y d z \\
& =\sum_{j=1}^{i} \sum_{k=1}^{\infty} \frac{1}{|B|} \int_{B} \int_{\left(2^{k+1} B\right) \backslash\left(2^{k} B\right)}\left|h_{t_{B}}(z, y)\left(b(y)-b_{B}\right) T_{j, 2}(f)(y)\right| d y d z \\
& \leq C \sum_{j=1}^{i} \sum_{k=1}^{\infty} 2^{k n} s\left(2^{(k-1) m}\right) \int_{2^{k+1} B}\left|\left(b(y)-b_{B}\right) T_{j, 2}(f)(y)\right| d y d z \\
& \leq C\|b\|_{*} \sum_{j=1}^{i} \sum_{k=1}^{\infty}(k+1) 2^{k n} s\left(2^{(k-1) m}\right) M_{r s}\left(T_{j, 2} f\right)(x) \\
& \leq C\|b\|_{*} \sum_{j=1}^{i} M_{r s}\left(T_{j, 2} f\right)(x) .
\end{aligned}
$$

Moreover, from case (1) it follows that

$$
I I I_{3} \leq C\|b\|_{*} \sum_{j=i+1}^{M} M_{r s}\left(T_{j, 2} f\right)(x) .
$$

So, $I I I \leq C\|b\|_{*} \sum_{j=1}^{M} M_{r s}\left(T_{j, 2} f\right)(x)$.

Combining the above estimates of $I, I I$ and $I I I$, we obtain (3.4).

From (3.4), we know that if $T$ is an operator satisfying (a), (b) and (c), then there exists $1<s<p$ such that $w \in A_{p / s}$ and

$$
M_{A}^{\sharp}(T f)(x) \leq C M_{S} f(x) .
$$

For the proof of (3.5), one can also see [12, Proposition 5.4]. Then combining (3.5), Lemmas 2.2 and 3.1, we have

$$
\|T f\|_{L^{p, \kappa}(w)} \leq C\left\|M_{A}^{\sharp}(T f)\right\|_{L^{p, \kappa}(w)} \leq\left\|M_{S} f\right\|_{L^{p, \kappa}(w)} \leq\|f\|_{L^{p, \kappa}(w)} .
$$


Combining this, (3.4), Lemmas 2.2 and 3.1, we have

$$
\left\|T_{b} f\right\|_{L^{p, \kappa}(w)} \leq C\left\|M_{A}^{\sharp}\left(T_{b} f\right)\right\|_{L^{p, \kappa}(w)} \leq \sum_{j=1}^{M} C\|b\|_{*}\left\|M_{r s}\left(T_{j, 2} f\right)\right\|_{L^{p, \kappa}(w)} \leq C\|b\|_{*}\|f\|_{L^{p, \kappa}(w)}
$$

for all $f \in L^{p, \kappa}(w)$. The proof of this theorem is completed.

Corollary 3.3 Let $T$ beoperators satisfying the above conditions (a), (b) and (c). Let $w \in A_{p}$, $1<p<\infty$. If $b \in B M O\left(\mathbb{R}^{n}\right)$, then there exists a constant $C$ such that

$$
\|[b, T] f\|_{L^{p, \kappa}(w)} \leq C\|b\|_{*}\|f\|_{L^{p, \kappa}(w)}
$$

for all $f \in L^{p, \kappa}(w)$.

\section{Competing interests}

The authors declare that they have no competing interests.

\section{Authors' contributions}

The authors completed the paper together. They also read and approved the final manuscript.

\section{Acknowledgements}

The authors would like to thank the referee for carefully reading the manuscript and for making several useful suggestions. This research was supported by the National Natural Science Foundation of China (Grant No. 11271092), Natural Science Foundation of Guangdong Province (Grant No. s2011010005367), Specialized Research Fund for the Doctoral Program of Higher Education (Grant No. 20114410110001, 20124410120002) and SRF of Guangzhou Education Bureau (Grant No. 2012A088)

Received: 4 July 2012 Accepted: 30 April 2013 Published: 17 May 2013

\section{References}

1. Morrey, CB: On the solutions of quasi-linear elliptic partial differential equations. Trans. Am. Math. Soc. 43, 126-166 (1938)

2. Chiarenza, F, Frasca, M: Morrey spaces and Hardy-Littlewood maximal function. Rend. Mat. Appl. 7, $273-279$ (1987)

3. Nakai, E: Hardy-Littlewood maximal operator, singular integral operators and the Riesz potentials on generalized Morrey spaces. Math. Nachr. 166, 95-103 (1994)

4. Palagachev, DK, Softova, LG: Singular integral operators, Morrey spaces and fine regularity of solutions to PDE's. Potential Anal. 20, 237-263 (2004)

5. Softova, L: Singular integrals and commutators in generalized Morrey spaces. Acta Math. Sin. Engl. Ser. 22, 757-766 (2006) (English summary)

6. Komori, Y, Shirai, S: Weighted Morrey spaces and a singular integral operator. Math. Nachr. 282, $219-231$ (2009)

7. Krantz, S, Li, S: Boundedness and compactness of integral operators on spaces of homogeneous type and applications, I. J. Math. Anal. Appl. 258, 629-641 (2001)

8. Lu, SZ, Mo, HX: Toeplitz-type operators on Lebesgue spaces. Acta Math. Sci., Ser. B, Engl. Ed. 29, 140-150 (2009)

9. Xie, PZ, Cao, GF: Toeplitz-type operators on homogeneous type spaces. Chin. Ann. Math., Ser. A 32, $219-228$ (2011) (Chinese)

10. Duong, XT, McIntosh, A: Singular integral operators with non-smooth kernels on irregular domains. Rev. Mat. Iberoam. 15, 233-265 (1999)

11. Stein, EM: Harmonic Analysis: Real Variable Methods, Orthogonality and Oscillatory Integrals. Princeton University Press, Princeton (1993)

12. Martell, JM: Sharp maximal functions associated with approximations of the identity in spaces of homogeneous type and applications. Stud. Math. 161, 113-145 (2004)

13. Burger, N: Espace des fonctions à variation moyenne bornée sur un espace de nature homogène. C. R. Acad. Sci. Paris, Sér. A-B 286, 139-141 (1978)

14. Bramanti, M, Cerutti, M: Commutators of singular integrals on homogeneous spaces. Boll. Unione Mat. Ital. 10 843-883 (1996)

15. Duong, XT, Yan, LX: Commutators of BMO functions and singular integral operators with non-smooth kernels. Bull. Aust. Math. Soc. 67, 187-200 (2003) 